Eisen, Mangan und freier Kohlensäure ein Urteil über die Brauchbarkeit von Wasser für Wasserleitungen gewonnen werden.

Dresden, im Januar 1905.

\title{
Verbesserter Apparat zur Milchfett-Bestimmung nach Gottlieb-Röse.
}

Von

\section{Armin Röhrig.}

Mitteilung aus der Chemischen Untersuchungsanstalt der Stadt Leipzig.

Von allen Verfahren der gewichtsanalytischen Fettbestimmung in der Milch, die in letzter Zeit bekannt geworden sind, einschließlich jener, die sich bereits ein Heimatrecht in der analytischen Praxis erworben haben, ist dem Gottlieb-Röse'schen wegen seiner einfachen und dabei exakten Ausführung der Vorzug zu geben.

Nachdem die Methodik dieses Verfahrens in neuerer Zeit durch die Arbeiten von Siegfeld ${ }^{1}$ ) und $\mathrm{Popp}^{2}$ ) einer eingehenden Durcharbeitung unterzogen worden ist, sodaß dasselbe nunmehr auf einer sicheren Basis beruht, ist die Gottlieb$R$ ös e'sche Methode unbestritten jene, die an analytischer Genauigkeit bei gleichzeitiger Schnelligkeit der Ausführung allen anderen überlegen ist.

Alle Laboratorien, die fortgesetzt eine große Anzahl von Milchuntersuchungen zu erledigen haben, werden nach solchen Methoden greifen müssen, die rasch und doch exakt arbeiten. Es ist kein $Z$ weifel darüber, daß erst durch die bekannte Gerber'sche acidbutyrometrische Bestimmung des Milchfettes, deren Hauptvorteil in einer raschen Ausführung liegt, die städtische Milchkontrolle zu jener Bedeutung gekommen ist, die sie im Rahmen der allgemeinen Nahrungsmittelkontrolle in größeren: Städten nunmehr einnimmt. Bis jetzt hat auch keine der neueren bekannt gewordenen Methoden der Gerber'schen den Rang streitig machen können. Bei aller Bequemlichkeit der Fettbestimmung nach Gerber wird doch der Nahrungsmittelchemiker nach anderen gewichtsanalytischen Methoden Umschau halten müssen, wenn es gilt, die gewonnenen Ergebnisse vor Gericht zu vertreten. Wenn auch der Gerber'schen Methode eine große Sicherheit bei richtiger Ausführung beizumessen ist, reicht sie unmöglich aus, solche Werte zu schaffen, auf die ein sachverständiges Gutachten sich allein stützen kann. Der gewissenhafte Nahrungsmittelchemiker wird stets bei ernsteren Beanstandungen bestrebt sein, die Resultate einer anderen und zwar gewichtsanalytischen Methode der Milchfettbestimmung herbeizuziehen und erst aus dem beiderseitigen Übereinstimmen der Ergebnisse zweier verschiedener Verfahren, deren Anwendbarkeit wissenschaftlich als einwandfrei gilt, die zur Abgabe eines Gutachtens notwendige und unerläßliche Sicherheit gewinnen.

Für solche Zwecke erweist sich nach meinen Erfahrungen in erster Linie die Gottlieb-Röse'sche Methode von allen bis jetzt bekannten als am zweckentsprechendsten.

1) Diese Zeitschrift $1903,6,259$.

) Diese Zeitschrift 1904, 7,6. 
Das Prinzip des Verfahrens ist, wie bekannt, sehr einfach. Eine abgewogene oder abgemessene Menge Milch wird mit einer nicht zu verdünnten Ammoniakflüssigkeit, Alkohol, Äther und Petroläther in der angegebenen Reihenfolge und in bestimmten Mengen unter jedesmaligem Durchschütteln versetzt. Die Äther-Petrolätherschicht enthält das gesamte Fett.

Nach der Gottlieb'schen Vorschrift, die auch von Siegfeld und Popp und anderen in ihren Versuchen eingehalten wurde, wird von der gesamten, das Fett enthaltenden Äther-Petrolätherlösung (etwa $50-52 \mathrm{ccm}$ ) alles bis auf $1,5 \mathrm{ccm}$, entsprechend $3 \%$, abgehebert und darin nach Verdunstung des Lösungsmittels das Fett bestimmt.

Die Apparatur besteht aus einem etwa $40 \mathrm{~cm}$ bohen in $1 / 2 \mathrm{~cm}$ geteilten Cylinder; von der abgetrennten ätherischen Schicht wird mit Hilfe einer Röhrenanordnung, ähnlich der einer Spritzflasche und eines kleinen Handgebläses, ein aliquoter Teil in ein Kölbchen gebracht.

Nach der alten Röse'schen Vorschrift ${ }^{1}$ ) erfolgte das Ausschütteln des Fettes aus der ammoniakaliseh-alkoholischen Milch mittels Äther-Benzin in einer großen Scheidebürette mit eingeschliffenem Stopfen und Glashahn. Von der abgelesenen Fettlösung sollte ein aliquoter Anteil abpipettiert und nach dem Verdunsten des Lösungsmittels der Fettgehalt auf die gesamte Fettlösung umgerechnet werden.

Gottlieb hat diese Vorschrift verlassen und den Cylinder mit Hebevorrichtung eingeführt, jedenfalls aus dem naheliegenden Grunde, die Metbode zu vereinfachen. In dem Abpipetieren von $10 \mathrm{ccm}$ Milch gegenüber dem Abwägen und Zurücklassen von 3\% der Äther-Petroläther-Fettlösung im Cylinder als Ausgleich für das Milchgewicht von $10 \mathrm{ccm}$ liegt eine Bequemlichkeit für den Analytiker, weil das exhaltene Ergebnis direkt Prozente an Fett ergiebt und ein nachträgliches Umrechnen erspart wird.

Als ich mich anschickte die Gottlieb-Röse'sche Methode der Fettbestimmung in unserer Anstalt einzuführen, ist es mir, wie wohl auch anderen Analytikern, ergangen. Trotz der naheliegenden und jeden Analytiker überzeugenden Vorteile ist die Methode nach der Gottlieb'schen Verbesserung unhandlich und nicht geeignet, sich die Gunst des Analytikers zu erwerben.

Auch Siegfeld ${ }^{2}$ ) schreibt hierüber:

${ }_{n}$ Die Methode hat auf den ersten Blick nichts Bestechendes. Die langen engen Cylinder sind unhandlich, schwer zu reinigen und leicht zu zerbrechen; das gleiche gilt in erhöhtem Maß3e von den langen dünnen Röhren, die zum Abziehen der Fettlösung in die Wägekölbchen dienen, und der Zusatz von 4 verschiedenen Reagentien ist umständlich. Sie hat aber vor der A d ams'schen den Vorzug, weniger Zeit und Arbeit zu erfordern; und die damit erzielten Ergebnisse sind recht zufriedenstellend."

Nur der umständlichen und unbandlichen Apparatur, die Gottlieb einführte, dürfte es zuzuschreiben sein, daß die $R \ddot{s}$ se'sche Metbode sich nicht allenthalben jener ungeteilten Anerkennung erfreut, die sie verdient.

Die von Gottlieb angegebenen Verbesserungen der alten Rös e'schen Methode dürften von wenigen als solche empfunden werden. Sie opfern die Bequemlichkeit und Handlichkeit des Apparates zugunsten eines geringen rechnerischen Vorteils.

1) Zeitschr. angew. Chem $1888,100$.

2) Diese Zeitschrift 1903, 6, 268. 
In der heutigen analytischen Praxis ist man aber bestrebt, die analytischen Methoden nach Möglichkeit zu vereinfachen.

Es war mir deshalb interessant, aus dem Jahresberichte des Hygienischen Institutes Hamburg ${ }^{1}$ ) zu ersehen, daß auch K. Farnsteiner den gerügten Mangel der Methode empfunden hat; denn er beschreibt ein Verfahren der Gottlieb-Röse'schen Methode, wobei ähnlich der früheren $\mathrm{R} o ̈ s e ' s c h e n$ Vorschrift eine besonders geeichte Bürette zur Anwendung kommt. Von der abgelesenen Äther-Petrolätherschicht wird wie dort ein aliquoter Anteil abpipettiert. Die nach diesem Verfahren gewonnenen Frgebnisse sind befriedigend.

Aber auch der Farnsteiner'schen wie der Röse'schen Bürette haftet noch ein Mangel an, der in dem lästigen Abpipettieren von ätherischen Lösungen liegt. Nur selten und bei großem persönlichen Geschicke wird es gelingen ein bestimmtes $\mathrm{MaB}$ an Äther.Petroläther aus einer Bürette mittels einer Pipette herauszunehmen und in ein Glasgefäf überzuführen. Diese Art des Abmessens gehört jedenfalls nicht zu den angenehmsten Laboratoriumsarbeiten, wenn sie häu* figer und rasch ausgeführt werden müssen, ganz abgesehen von den möglichen Fehlern, die in der Ausdehnungsfähigkeit des Äther-Petroläthers liegen. Zur Vermeidung solcher Arbeiten, die von den gewöhnlich stark beschäftigten Assistenten immer unangenehm empfunden werden, have ich in Anlehnung an die früheren $R$ ö se'schen Angaben einen Apparat zusammengestellt, der das Abpipettieren überflüssig macht und dem Gottlieb-Röse'schen Verfahren jene Handlichkeit gibt, die zu einem häufigen und bequemen Gebrauche wünschenswert ist.

Nach einjährigem praktischem Gebrauche hat sich dieser Apparat vollauf bewährt; seine Fehlerquellen sind bei richtiger Anwendung nicht erheblich und ein großer Vorzug liegt darin, daß eine allzu peinliche Vorsicht beim Beschicken und Ablesen überflüssig ist. Auch Röse hat bereits darauf hingewiesen, daß es beim Abmessen von Alkohol und Wasser, das er der Milch noch zusetzte, auf irgendwelche Genauigkeit nicht ankommt. Nach M. Popp gilt dies auch für das Ammoniak und ganz selbstverständlich anch für die fettlösenden Flüssigkeiten.

Die Abbildung des Apparates (Fig. 8) zeigt seine Einzelheiten

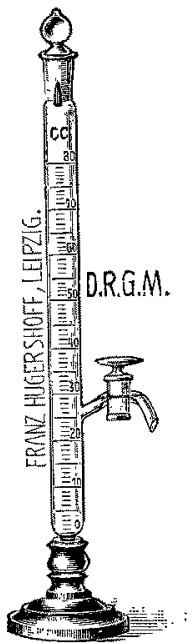

Fig. 8. und bedarf derselbe keiner besonderen Beschreibung.

Die Ausführung einer Milchfettbestimmung mit diesem Apparate geschieht auf folgende Weise:

„Von der durchgeschüttelten Milch (es kann auch bereits geronnene sein) pipettiert man $10 \mathrm{ccm}$ in den gereinigten Apparat, versetzt mit $2 \mathrm{ccm}$ starkem Ammoniak, sodann mit $10 \mathrm{ccm}$ gewöhnlichem Alkobol (etwa 90\%-igem) und schüttelt jedesmal kräftig um. Sodann fügt man nacheinander $25 \mathrm{ccm}$ Äther und $25 \mathrm{ccm}$ niedrig siedenden völlig flüchtigen Petroläther hinzu und schüttelt jedesmal lä̈ftig um. Unterdessen hat man eine Glasschale (wir verwenden solche mit flachem Boden) oder ein Becherglas getrocknet und tariert. Von der abgelesenen und notierten, etwa 1 Stunde der Ruhe überlassenen Äther-Perolätherschicht läßt man durch den Hahn $30 \mathrm{ccm}$ ab, verdunstet bei $100^{\circ}$, trocknet und wägt. Das erhaltene Gewicht berechnet man auf

1) Jahresbericht des Hygienischen Instituts Hamburg 1900-1902, S. 25-27; vergl. diese Zeitschrift 1904, 7, 105. 
die ganze Fettlösung und dividiert die Fettmenge durch das spezifische Gewicht der Mileh."

Eine Fehlerquelle des Verfahrens, auf welche auch schon $\mathrm{K}$. F a rnsteiner hingewiesen hat, liegt in dem Abpipettieren der Milch, weil selbst aus amtlich geeichten Pipetten das Gewicht von $10 \mathrm{cem}$ Milch nicht dem entspricht, welches ihm nach dem spezifischen Gewichte zukommt.

Von einer großen Avzahl von Beobachtungen führe ich einige Gewichte von $10 \mathrm{com}$ Milch an, wie sie in den Apparat verbracht wurden:

\begin{tabular}{c|c|c|c|c|c}
\hline $\begin{array}{c}\text { Spezif. Gewicht } \\
\text { der Milch } \\
\text { bei } 15^{0} \mathrm{C}\end{array}$ & $\begin{array}{c}10 \mathrm{cem} \text {, aus der } \\
\text { Pipette abge- } \\
\text { lassen, wiegen } \\
\mathrm{g}\end{array}$ & Differenz & $\begin{array}{c}\text { Spezif. Gewieht } \\
\text { der Mileh } \\
\text { bei } 15^{0} \mathrm{C}\end{array}$ & $\begin{array}{c}\text { 10 ecm, aus der } \\
\text { Pipette abge- } \\
\text { lassen, wiegen } \\
\mathrm{g}\end{array}$ & Differenz \\
\hline 1,0283 & 10,2000 & 0,0830 & 1,0278 & 10,1980 & $\mathrm{~g}$ \\
1,0289 & 10,2402 & 0,0490 & 1,0270 & 10,2064 & 0,0800 \\
1,0268 & 10,1880 & 0,0800 & 1,0291 & 10,1852 & 0,1058 \\
1,0267 & 10,1816 & 0,0854 & 1,0279 & 10,1742 & 0,1050 \\
1,0257 & 10,1870 & 0,0700 & 1,0263 & 10,1720 & 0,0910
\end{tabular}

Diese Abweichungen, so erheblich sie auch erscheinen, betragen doch nie mehr als $0,1 \mathrm{~g}$, also etwa $1 \%$ der Einwage, bei einer normalen Milch mit $3 \%$ Fett also einem Verluste von 0,03\% Fett entsprechend. Wollte man etwa deshalb das Abpipettieren durch das genauere Abwägen der Milch ersetzen, so gäbe man einen nicht unbeachtlichen Vorteil des ganzen Verfahrens preis.

Die weiterhin angeführten Ergebnisse lassen auch deutlich erkennen, daß noch irgendwelche andere Fehler außerbalb des Apparates sich geltend machen, eine Erscheinung, auf welche auch Siegfeld und Popp bereits hingewiesen haben.

Auch unsere Beobachtungen bestätigen, daß zunächst Fehler in der Art des Trocknens und im Abwägen der nach dem Verdunsten der Ätherfettlösung verbleibenden Fettmenge vorkommen können. Vermeidet man ein allzulanges und scharfes Trocknen und gewöhnt man sich daran, erst nach völligem Abkühlen zu wägen, so gehen die Fehler um ein geringes zurück und gleichen sich, wie die gewonnenen Ergebnisse beweisen, sogar gegenseitig aus.

Immerhin war es wichtig mit Hilfe desselben Apparates den Unterschied einmal festzustellen, der in der Beschickung des Apparates einerseits durch Wägung, andererseits durch Pipettierung der Milch verursacht wird.

Die dahin zielenden Ergebnisse waren folgende:

\begin{tabular}{|c|c|c|c|c|}
\hline \multirow{2}{*}{ No. } & \multirow{2}{*}{\multicolumn{2}{|c|}{ Nach Gerber }} & \multicolumn{2}{|c|}{ Nach Gottlieb-Röse } \\
\hline & & & gewogen & gemessen \\
\hline 1 & $\left\{\begin{array}{l}4,45 \\
4,50\end{array}\right\}$ & Mittel $4,475 \%$ & $4,430 \%$ & $4,426 \%$ \\
\hline 2 & $\left.\begin{array}{l}2,40 \\
2,42\end{array}\right\}$ & " 2,410, & 2,430 & 2,429 \\
\hline
\end{tabular}

Diese beiden Versuche haben mich vollständig überzeugt, daß der geringe Gewichtsunterschied, der im Abpipettieren der Milch gegenüber dem Abwägen von genau $10 \mathrm{~g}$ liegt, auf das Endergebnis keinen wesentlichen oder doch keinen bestimmenden Einflu@ hat. Dies ist auch bereits hinlänglich bekannt und wird durch die von Gottlieb angegebenen Änderungen der Methode $R o ̈ s e$, die von vielen nachgeprüft 
wurde, bestätigt. Obwohl nicht jede Milch ein spezifisches Gewicht von 1,030 besitzt, sind die nach Gottlieb gewonnenen Ergebnisse unter dieser Annahme durchaus brauchbare. Der Fehler liegt noch unter $2 \%$ und kommt nicht zum Ausdrucke.

Die nachstehend zusammengestellten Ergebnisse sind aus unseren Büchern ohne Weglassung eines einzigen ausgezogen worden. Sie dürften nicht allenthalben den Leistungen entsprechen, zu denen der Apparat bei einigermaßen sorgsamerer Behandlung fähig ist. Man muß berücksichtigen, daß 4 verschiedene Analytiker den ihnen bis dahin unbekannten Apparat ohne Vorübung haben bedienen müssen, und daß denselben auch keine Zeit zur Wiederholung von etwas stark abweichenden Analysen zur Verfügung stand. Auch darf nicht unberücksichtigt bleiben, daß die nach Gerber gewonnenen Vergleichswerte Mittelwerte zweier Bestimmungen sind; während nach Gottlieb-Röse immer nur eine Analyse ausgeführt wurde.

Es wurde an Fett gefunden:

\begin{tabular}{|c|c|c|c|c|c|c|c|c|c|c|}
\hline \multicolumn{2}{|c|}{$\begin{array}{l}\text { Nach } \\
\text { Gerbor }\end{array}$} & $\begin{array}{c}\text { Nach } \\
\text { Gottlieb- } \\
\text { Rose }\end{array}$ & \multirow{2}{*}{$\begin{array}{c}\text { Differenz } \\
\text { nach Gott- } \\
\text { lieb-Rose } \\
\begin{array}{c}\text { gegenuber } \\
\text { Gerbor }\end{array} \\
+0,04\end{array}$} & \multicolumn{2}{|c|}{$\begin{array}{c}\text { Nach } \\
\text { Gerber }\end{array}$} & $\begin{array}{c}\text { Nach } \\
\text { Gottlieb } \\
\text { Röse }\end{array}$ & \multirow{2}{*}{$\frac{\begin{array}{c}\text { Differenz } \\
\text { naeh Gott- } \\
\text { lieb-Rose } \\
\text { gegenuber } \\
\text { Gerber }\end{array}}{+0,05}$} & $\begin{array}{c}\text { Nach } \\
\text { Qexbex }\end{array}$ & $\begin{array}{c}\text { Nach } \\
\text { gottieb } \\
\text { Rose }\end{array}$ & \multirow{2}{*}{$\begin{array}{c}\begin{array}{c}\text { Differenz } \\
\text { nach Gott- } \\
\text { lieb-Rose } \\
\text { gegenuber } \\
\text { Gerber }\end{array} \\
\mid \begin{array}{l}\text { t } 0,09\end{array}\end{array}$} \\
\hline $2,90 \quad 0$ & $\%$ & $2,94^{\circ} / 0$ & & 2,30 & & $2,35 \%$ & & 2,80 & $2,89 \%$ & \\
\hline 3,10 & & 3,06 . & $-0,04$ & 2,325 & " & 2,32 & $-0,005$ & 2,80 & 2,73 , & $-0,07$ \\
\hline 2,775 & $n$ & 2,77 * & $-0,005$ & 2,95 & 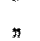 & 2,92 & $-0,03$ & 2,90 & 2,76 & $-0,14$ \\
\hline 2,85 & $\pi$ & 2,90 * & $\div 0,05$ & 2,55 & $\pi$ & 2,55 & 0 & 2,95 & 2,87 & $-0,08$ \\
\hline 2,55 & $n$ & 2,55 , & 0 & 2,32 & $"$ & 2,32 & 0 & 2,225 & 2,11, & $-0,115$ \\
\hline 2,70 & 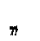 & 2,65 , & $-0,05$ & 2,95 & " & $2,97 \pi$ & $+0,02$ & 2,92 & 2,87 n & $-0,05$ \\
\hline 2,75 & , & 2,68 , & $-0,07$ & 2,20 & $\pi$ & 2,36 & $+0,16$ & 2,89 & 2,85 & $-0,04$ \\
\hline 2,725 & $n$ & 2,67 , & $-0,055$ & 2,55 & $n$ & 2,57 , & $+0,02$ & 2,225 & 2,20 & $-0,025$ \\
\hline 3,32 & $n$ & 3,31 ; & $-0,01$ & 2,65 & $\pi$ & 2,64 , & $-0,01$ & 2,80 & 2,78 & $-0,02$ \\
\hline 1,75 & " & 1,76 & $+0,01$ & 3,45 & $n$ & 3,42 , & $-0,03$ & 2,75 & 2,79 , & $+0,04$ \\
\hline 3,47 & , & 3,48 & $+0,01$ & 2,825 & " & 2,86 , & $+0,035$ & 2,675 & $2,65 \pi$ & $-0,025$ \\
\hline 2,30 & $\bar{\pi}$ & 2,39 & $+0,09$ & 2,60 & \# & 2,66 * & $+0,06$ & 2,50 & 2,46 , & $-0,04$ \\
\hline 4,60 & , & 4,73 & $+0,13$ & 2,70 & $n$ & 2,72 & $+0,02$ & 2,875 & 2,84 , & $-0,035$ \\
\hline 2,29 & 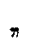 & 2,37 & $+0,08$ & 2,49 & 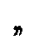 & 2,49 & 0 & 2,85 & 2,81 , & $-0,04$ \\
\hline 2,70 & $n$ & 2,80 & $+0,10$ & 2,375 & $\pi$ & 2,30 * & $-0,075$ & 2,825 & 2,78 , & $-0,045$ \\
\hline 2,925 & $n$ & 2.94 & $+0,015$ & 2,27 & $n$ & 2,55 & $-0,02$ & 3,30 & 3,30 , & 0 \\
\hline 2,50 & $n$ & 2,55 & $+0,05$ & 2,85 & 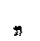 & 2,84 & $-0,01$ & 2,90 & 2,92 * & $+0,02$ \\
\hline 2,975 & त & 2,98 , & $+0,005$ & 2,65 & , & 2,62 & $-0,08$ & 2,38 & 2,30 & $-0,08$ \\
\hline 2,50 & 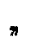 & 2,54 & $+0,04$ & 2,65 & , & 2,65 , & 0 & 2,70 & 2,70 & 0 \\
\hline 2,13 & $\pi$ & 2,19 , & $+0,06$ & 2,975 & $\pi$ & 2,97 , & $-0,005$ & 2,675 & 2,70 & $+0,025$ \\
\hline 2,235 & $\pi$ & 2,23 , & $-0,005$ & 3,00 & $n$ & 2,97 , & $-0,03$ & 2,80 & 2,74 , & $-0,06$ \\
\hline 2,55 & 3 & 2,51 & $-0,04$ & 2,35 & " & 2,34 * & $-0,01$ & 2,80 & 2,71 & $-0,09$ \\
\hline 2,775 & 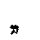 & 2,76 & $-0,015$ & 2,20 & $\pi$ & 2,19 & $-0,01$ & 2,325 & $2,33 *$ & $+0,005$ \\
\hline 2,30 & $\pi$ & 2,26 , & $-0,04$ & 2,60 & n & 2,59 , & $-0,01$ & 2,80 & 2,79 , & $-0,01$ \\
\hline 2,40 & 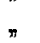 & 2,37 & $-0,03$ & 2,375 & $n$ & 2,20 , & $-0,175$ & 2,80 & 2,72 & $-0,08$ \\
\hline 2,70 & $\pi$ & 2,67 & $-0,03$ & 3,25 & $n$ & 3,15 n & $-0,10$ & 2,875 & 2,76 , & $-0,115$ \\
\hline 2,59 & $\pi$ & 2,59 & 0 & 2,60 & \# & 2,58 , & $-0,02$ & 2,30 & 2,28 , & $-0,02$ \\
\hline 2,98 & za & $2,98 \pi$ & 0 & 2,75 & $n$ & 2,71 , & $-0,04$ & 2,83 & 2,79 & $-0,04$ \\
\hline 2,70 & $\pi$ & 2,65 , & $-0,05$ & 2,40 & $n$ & 2,43 & $+0,03$ & 2,00 & 2,01, & $+0,01$ \\
\hline 2,35 & $\pi$ & 2,38 & $+0,03$ & 2,95 & $\pi$ & 2,94 , & $-0,01$ & 2,30 & 2,26 , & $-0,04$ \\
\hline 2,50 & $\pi$ & 2,46 , & $-0,04$ & 2,875 & $r$ & 2,78 n & $-0,095$ & 2,25 & 2,30 , & $+0,05$ \\
\hline 2,00 & $\pi$ & $1,99_{n}$ & $-0,01$ & 2,875 & $n$ & 2,82 & $-0,055$ & 2,475 & 2,45 & $-0,025$ \\
\hline
\end{tabular}




\begin{tabular}{|c|c|c|c|c|c|c|c|c|c|c|}
\hline $\begin{array}{c}\text { Nach } \\
\text { Gerber }\end{array}$ & $\begin{array}{c}\text { Nach } \\
\text { Gottlieb- } \\
\text { Röse }\end{array}$ & $\begin{array}{c}\text { Differenz } \\
\text { nach Gott- } \\
\text { lieb-Rös } \\
\text { gegenüber } \\
\text { Gerber }\end{array}$ & \multicolumn{2}{|c|}{$\begin{array}{c}\text { Nach } \\
\text { Gerber }\end{array}$} & $\left\{\begin{array}{c}\text { Nach } \\
\text { Gottieb- } \\
\text { Rose }\end{array}\right.$ & $\begin{array}{c}\text { Differenz } \\
\text { nach Gott- } \\
\text { lieb-Röse } \\
\text { gegenuber } \\
\text { Ge rber }\end{array}$ & \multicolumn{2}{|c|}{$\begin{array}{l}\text { Nath } \\
\text { Gerber }\end{array}$} & $\begin{array}{c}\text { Nach } \\
\text { Gottlieb } \\
\text { Rose }\end{array}$ & $\begin{array}{c}\text { Differenz } \\
\text { nach Gott- } \\
\text { lieb-Röse- } \\
\text { gegenüber } \\
\text { Gerber }\end{array}$ \\
\hline $2,500 \%$ & $2,47{ }^{\circ}, 0$ & $-0,03$ & $2,875^{\circ}$ & & $2,94^{\%} / 0$ & $+0,065$ & 2,50 & $\%$ & $2,47 \%$ & $-0,03$ \\
\hline 2,525 & 2,58 & $+0,055$ & 2,60 & & 2,62 & $+0,02$ & 3,07 & $n$ & 3,10 & +0.03 \\
\hline 2,90 & 2,88 & $-0,02$ & 2,85 & & 2,82 & $-0,03$ & 2,95 & n & 2,93 & $-0,02$ \\
\hline 2,05 & 2,04 & $-0,01$ & 2,60 & & 2,62 & $+0,02$ & 3,40 & n & $3,375_{n}$ & $-0,025$ \\
\hline 2,70 & $2,65 n$ & $-0,05$ & 2,725 & & 2,70 & $-0,025$ & 2,25 & $n$ & 2,29 & $+0,04$ \\
\hline 2,50 & 2,48 & $-0,02$ & 3,275 & & 3,28 " & $+0,005$ & 2,95 & n & 2,75 & $-0,20$ \\
\hline 2,825 & 2,80 & $-0,025$ & 2,55 & & 2,57 & $+0,02$ & 3,05 & \# & 2,98 n & $-0,07$ \\
\hline 2,65 & 2,61 & $-0,04$ & 2,67 & & 2,65 & $-0,02$ & 2,70 & n & 2,66 & $-0,04$ \\
\hline 1,975 & 1,98 & $+0,005$ & 2,05 & $"$ & 2,07 & +0.02 & 2,50 & 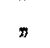 & $2,55 \pi$ & $+0,05$ \\
\hline 2,57 & 2,43 & -0.14 & 2,75 & " & 2,79 & $+0,04$ & 2,60 & $n$ & 2,61 n & $+0,01$ \\
\hline 2,50 & $2,52 \pi$ & $+0,02$ & 2,50 & $n$ & 2,46 . & $-0,04$ & 2,40 & $n$ & 2,33 & $-0,07$ \\
\hline 3,34 & 3,34 & 0 & 2,80 & " & $2,75 n$ & $-0,05$ & 2,50 & $n$ & 2,51 , & $+0,01$ \\
\hline 2,625 & 2,51 " & $-0,115$ & 2,85 & \# & 2,78 & $-0,07$ & 2,80 & n & 2,75 & $-0,05$ \\
\hline 2,40 & 2,37 & $-0,03$ & 2,90 & " & 2,88 , & $-0,02$ & 2,80 & $n$ & 2,74 , & $-0,06$ \\
\hline 2,50 & 2,51 & $+0,01$ & 2,40 & 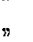 & 2,38 & $-0,02$ & 2,40 & , & 2,43 & $+0,03$ \\
\hline 2,45 & 2,46 & $+0,01$ & 2,90 & \# & 2,84 & $-0,06$ & 2,40 & 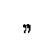 & 2,49 " & $+0,09$ \\
\hline 2,325 & 2,33 & $+0,005$ & 2,78 & " & 2,75 , & $-0,03$ & 2,70 & $n$ & 2,64 , & $-0,06$ \\
\hline
\end{tabular}

Die Ergebnisse sind im Vergleich mit anderen wesentlich umständlicheren Verfahren im Dienste der täglichen Milchkontrolle, die eine rasche Erledigung erfordert, als brauchbare $z u$ bezeichnen. Von einer eingehenderen Besprechung der mit dem neuen Apparate gewonnenen Ergebnisse glaube ich absehen zu können. Daß das Verfahren gegenüber allen anderen gewichtsanalytischen Methoden auch das billigste ist, mag nebenbei noch erwähnt werden.

Unter Berücksichtigung der in den Gottlieb'schen Verbesserungen des Röseschen Verfahrens liegenden Motive läßt sich überdies das Verfahren noch weiterhin vereinfachen. Zum Ausgleiche der Gewichtsdifferenz der gewogenen gegenüber der pipettierten Milch kann man eine neue Pipette herstellen, die rund 9,7 cem Milch entsprechend $10 \mathrm{~g}$ unter Annahme eines mittleren spezifischen Gewichtes von 1,03 dem Apparate zuführt. Wenn man dann noch genau die Hälfte der Äther-Petrolätherfettlösung: abläßt und verdunstet, so erhält man den Fettgehalt der Milch durch Multiplikation des Verdunstungsrückstandes mit 2. Bei dem von mir eingehaltenen Verfahren habe ich von einer solchen Abänderung vorerst abgesehen, weil mir das spezifische Gewicht jeder untersuchten Milch bekannt war und ich zunächst den Apparat unter Vermeidung von möglichen Fehlerquellen erproben wollte. Für häufig auszuführende Kontrollbestimmungen in milchwirtschaftlichen Instituten, wenn eine über $3 \%$ hinausgehende Genauigkeit nicht notwendig ist, wird man jede Vereinfachung des Analysen- und Rechenwerkes jedoch willkommen heißen. Die vereinfachte Methode würde sich dann etwa folgendermaßen gestalten:

„Man pipettiert 9,7 ccm Milch in die Stehbürette, versetzt mittels eines Automaten, wie er zum Abmessen von Amylalkohol oder Schwefelsäure bei dem Gerber'schen Verfahren jetzt gebräuchlich ist, mit $2 \mathrm{cem}$ starkem Ammoniak, $10 \mathrm{ccm}$ Alkohol $(90 \%)$, mischt durch Umschwenken, fügt unter jedesmaligem Umschütteln je $25 \mathrm{ccm}$ Äther und $25 \mathrm{ccm}$ niedrig siedenden Petroläther hinzu. Nach völligem Absetzen 
(nach etwa 1 Stunde) läßt man die Hälfte der Äther-Petrolätherschicht in eine gewogene Schale (Becherglas) ablaufen und verdunstet das Lösungsmittel. Nach kurzem Trocknen ergibt das mit 20 multiplizierte Gewicht des zurückbleibenden Fettes direkt die Prozente an Milchfett."

Einige nach diesem Verfahren gewonnene Ergebnisse mögen hier angeführt werden:

$\begin{array}{ccccc}\text { No. Spez. Gewicht } & \begin{array}{c}\text { Fett nach } \\ \text { Geiber } \\ \text { bei C }\end{array} & \begin{array}{c}\text { Fett nach } \\ \text { (Mittel) }\end{array} & \begin{array}{c}\text { Differenz nach } \\ \text { bei } 9,7 \text { cem Einwage }\end{array} \\ \begin{array}{ccc}\text { Gottlieb gegen- } \\ \text { ibber Gerber }\end{array} \\ 1 & 1,0311 & 2,70 \% & 2,70 \% & 0 \\ 2 & 1,0279 & 2,60 \% & 2,64 \% & +0,04 \mathrm{~g} \\ 3 & 1,0300 & 4,60 \% & 4,65 \% & +0,05 \% \\ 4 & 1,0341 & 2,70 \% & 2,72 \% & +0,02 \%\end{array}$

Für die Untersuchung des Fettgehaltes in Rahm ist der Apparat, wie nachstehende Ergebnisse beweisen, in gleicher Weise verwendbar. Man wird jedoch in diesem Falle die zu prüfende Menge einwägen und das an $10 \mathrm{cem}$ fehlende Wasser ergänzen.

Folgende Ergebnisse sind so erhalten worlen:

\begin{tabular}{|c|c|c|c|c|c|}
\hline No. & Bezeichnung & & $\begin{array}{c}\text { Fett } \\
\text { nach Gerber }\end{array}$ & $\begin{array}{c}\text { Fett nach } \\
\text { Gottlieb-Röse }\end{array}$ & $\begin{array}{l}\text { Differenz nach } \\
\text { Gottlieb gegen- } \\
\text { über Gerber }\end{array}$ \\
\hline 1) & Saure Sahne & . & $22,00 \%$ & $22,06 \%$ & $+0,06 \%$ \\
\hline 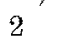 & " & . & 15,50 , & $15,82 \pi$ & $+0,32$ \\
\hline 8 & Süße Salne & & 13,50 & 13,64 n & $+0,14$ \\
\hline 4 & Kaffeesahne & . & 5,40 & 5,39 & $-0,01$ \\
\hline
\end{tabular}

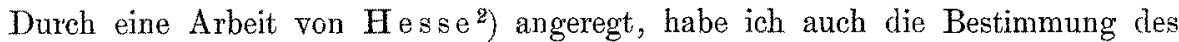
Fettes in Butter mit demselben Apparate ausgeführt, die mich zu weiteren Versuchen ermutigen, zumal alle bis jetzt bekannten Verfahren zur Erlangung befriedigender Ergebnisse eine besondere Schulung voraussetzen, andererseits wegen des erforderlichen Zeit- und Materialaufwandes als zu kostspielig gelten.

Das einzuhaltende Verfahren ist etwa folgendes:

Man verreibt die auf etwa $30^{\circ} \mathrm{C}$ erwärmte Butter in einem Mörser oder Becherglase möglichst gleichmäßig, entnimmt mittels eines $5 \mathrm{~cm}$ langen, an beiden Seiten offenen und vorher tarierten Glasröhrchens eine Stichprobe und stellt durch nochmaliges Wägen die Buttermenge fest. Die mit samt dem Gläschen in den Apparat gebrachte Butter erweicht man durch Einhalten des Apparates in ein warmes Wasserbad, oder versetzt mit $10 \mathrm{cem}$ heißem Wasser, dann wieder mit Ammoniak, Alkohol, Äther und Petroläther, wie dies bei der Milch beschrieben, läßt nach völligem Absetzen wiederum $30 \mathrm{ccm}$ der Fettlösung $a b$ und verfährt im übrigen genau wie bei der Fettbestimmung in der Milch.

Um die Brauchbarkeit des Apparates auch für diesen Zweck zu beweisen, führe ich einige weitere Fettbestimmungen an, die nach der Soxhlet'schen Extraktionsmethode kontrolliert worden sind:

Fett nach $\left\{\begin{array}{lllllll}\text { Soxhlet . . } & 88,95 & 84,82 & 84,39 & 81,51 & 76,80 & 80,39 \% \\ \text { Gottlieb.Röse } & 84,10 & 84,86 & 84,40 & 81,31 & 76,73 & 79,90 \%\end{array}\right.$

1) No. 1, 2 und 3 wurden im Verbältnis $1: 5$ mit Wasser verdünnt; alle 4 Proben sind nach der vereinfachten Methode $(9,7 \mathrm{~cm}$ Einwage) untersucht worden.

2) Diese Zeitschrift 1904, 8, 673 . 
Abgesehen von der Dauer der Abwägung beansprucht die Ausführung der Fettbestimmung in der Butter genau dieselbe Zeit, wie eine Milchfettbestimmung. Die Trennungsschicht beider Flüssigkeiten ist so scharf, daß eine genaue und bequeme Ablesung möglich ist.

Die Firma Franz Hugershof in Leipzig hat die Herstellung und den Vertrieb des Apparates, der dieser Firma durch Deutsches Reichsgebrauchsmuster geschützt wurde, übernommen.

\section{Referate.}

\section{Allgemeine Bestandteile der Nahrungs- und Genußmittel.}

A. Kossel und D. Dakin: Beiträge zum System der einfachsten Eiw eißkörper. (Zeitschr. physiol. Chem. 1903/4, 40, 565-571). - Verff. haben Untersuchungen angestellt, die zeigen, daß der Aminovaleriansäure und dem Serin eine weitere Verbreitung unter den Protaminen zukommt. So haben sie zunächst über einige Spaltungsprodukte des Salmins Studien gemacht. Als Ausgangsmaterial dienten reife Lachstestikel, welche nach dem Verfahren von A. Kossel auf Protaminsulfat verarbeitet wurden. Nach ihren Untersuchungen zerfällt das Salmin in mindestens vier verschiedenartige Atomgruppen: Arginin, Pyrrolidinkarbonsäure, Aminovaleriansäure und Serin. In ähnlicher Weise wurde das Serin aus Clupein dargestellt. Im Clupein sind hiernach bisher nachgewiesen: Arginin, Aminovaleriansäure und Serin. Verff. haben alsdann Untersucbungen über die Protamine des Karpfenspermas angestellt. Sie untersuchten Karpfentestikel verschiedener Herkunft und zu verschiedenen Jahreszeiten. Es ergab sich, daß in den Karpfentestikeln mindestens zwei verschiedenartige Protamine vorhanden sind, deren eines ( $\alpha$-Cyprinin) einer neuen Klasse von Eiweißsubstanzen angehört und von den bisher bekannten Protaminen ebenso verschieden ist, wie von den Histonen. Max Milller.

Th. Gimbel: Uber die Verteilung des Stickstoffes im Eiweißmolekül. (Beitr. zur chem. Physiol, und Pathol. 1904, 5, 297-312.) - Unter obigem Namen hat W. Hausmann (Z. 1899, 2, 657 und 1900, 3, 615) ein Verfahren ausgearbeitet, das es ermöglichen sollte, auf wenig umständliche Art und mit einer geringen Menge Ausgangsmaterial einen Überblick über die Bindung des Stickstoffs im Eiweiß zu gewinnen. Das von ihm geübte Verfahren setzt sich aus nachstehenden Einzeloperationen zusammen: 1. Spaitung von etwa $1 \mathrm{~g}$ des Eiweißkörpers mit siedender konzentrierter Salzsäure. 2. Bestimmung des dabei in Ammoniumsalz übergeführten Stickstoffs durch Abdestillation mit Magnesia (Amidstickstoff, Ammoniakstickstoff, leicht abspaltbarer Stickstoff). 3. Fällung der ammoniakfrei gemachten Flüssigkeit mit Phosphorwolframsäure und Bestimmung des in den Niederschlag eingegangenen Stickstoffs (Diaminostickstoff, basischer Stickstoff). 4. Bestimmung des durch Magnesia nicht austreibbaren und durch Phosphorwolframsäure nicht fällbaren Stickstoffs (Monoaminostickstoff). Verf. hat nun dieses Hausmann'sche Verfahren einer Nachprüfung unterzogen und dabei gefunden, daß es für Amidstickstoff sehr scharfe, für den Monoaminostickstoff annähernd genaue, für den Diaminostickstoff bis zu $0,8 \%$ schwankende, meist zu niedrige Werte gibt. Dieser Umstand berechtigt zu der Frage, ob dem Verfahren überhaupt ein Wert für den von Haus mann ins Auge gefaften Zweck, die Charakterisierung der Proteinstoffe, zulkommt. Nun sind die gewomnenen Amidzahlen sehr zuverlässig und auch die Monoaminowerte sind trotz der ihnen anhaftenden Fehler genau genug, um eine Vorstellung über den Gehalt eines 